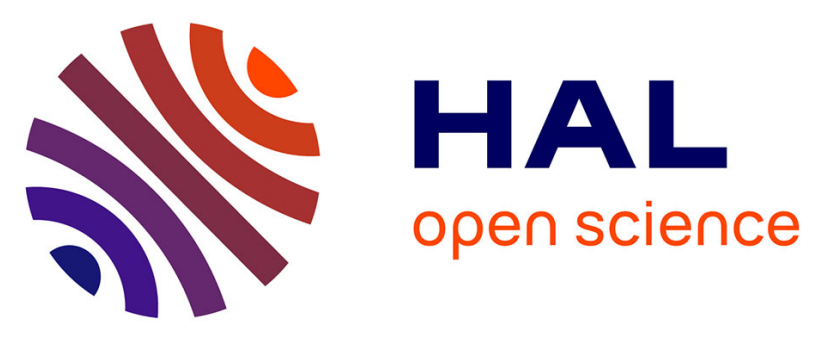

\title{
Critères d'indications de la biopsie rénale chez les patients diabétiques de type 2 protéinuriques: enquête auprès des néphrologues français
}

Jonathan Maurice Chemouny, Aurélie Sannier, Guillaume Hanouna, Quentin Raimbourg, Eric Daugas, Cécile Vigneau, François Vrtovnsik

\section{To cite this version:}

Jonathan Maurice Chemouny, Aurélie Sannier, Guillaume Hanouna, Quentin Raimbourg, Eric Daugas, et al.. Critères d'indications de la biopsie rénale chez les patients diabétiques de type 2 protéinuriques: enquête auprès des néphrologues français. Néphrologie \& Thérapeutique, 2019, 15 (7), pp.524-531. 10.1016/j.nephro.2019.05.004 . hal-02396569

HAL Id: hal-02396569

https://hal-univ-rennes1.archives-ouvertes.fr/hal-02396569

Submitted on 11 Dec 2019

HAL is a multi-disciplinary open access archive for the deposit and dissemination of scientific research documents, whether they are published or not. The documents may come from teaching and research institutions in France or abroad, or from public or private research centers.
L'archive ouverte pluridisciplinaire HAL, est destinée au dépôt et à la diffusion de documents scientifiques de niveau recherche, publiés ou non, émanant des établissements d'enseignement et de recherche français ou étrangers, des laboratoires publics ou privés. 
Critères d'indications de la biopsie rénale chez les patients diabétiques de type 2 protéinuriques : Enquête auprès des néphrologues français

\section{Criteria to indicate Kidney biopsy in type 2 diabetic patients with proteinuria: Survey among French nephrologists}

Jonathan M Chemouny, ${ }^{1,2,3}$ Aurélie Sannier, ${ }^{4,5}$ Guillaume Hanouna, ${ }^{5,6}$ Quentin Raimbourg, ${ }^{6}$ Eric Daugas, ${ }^{5,6,7}$ Cécile Vigneau, ${ }^{1,2,8}$ Francois Vrtovnsik..$^{5,6,7}$

1 CHU Rennes, Service de Néphrologie, Rennes, France

2 Université Rennes1, Faculté de médecine, Rennes, France

3 Institut Micalis, INRA, AgroParisTech, University Paris-Saclay, Jouy-en-Josas, France

4 Laboratoire d'anatomopathologie et de cytologie, Hôpital Bichat-Claude Bernard, AP-HP, Paris, France

5 Université Paris Diderot, Sorbonne Paris Cité, Paris 75018, France

6 Service de néphrologie, Hôpital Bichat-Claude Bernard, AP-HP, DHU Fire, Paris 75018 Paris

7 INSERM 1149, Center for Research on Inflammation (CRI), Paris, France

8 IRSET - UMR 1085, Rennes, France

\section{Correspondance à adresser à:}

Dr. Jonathan Chemouny,

Service de néphrologie,

Hôpital Pontchaillou,

2 rue Henri Le Guilloux,

35033 Rennes cedex 9,

France;

Tel +33 2992843 96Fax: +33299284150

e-mail: jonathan.chemouny@chu-rennes.fr

Titre courant: BR dans le DT2 : enquête 


\section{RESUME}

Le diagnostic de néphropathie diabétique est généralement clinique. Une biopsie rénale peut être réalisée en cas d'atypie pour éliminer une maladie rénale non diabétique. Nous avons évalué l'opinion des néphrologues francophones par un questionnaire anonyme portant sur les critères cliniques et biologiques - en particulier l'absence de rétinopathie diabétique, la présence d'une hématurie, la diminution du DFG ou l'élévation de la protéinurie - les amenant à retenir l'indication de biopsie rénale chez des patients diabétiques de type 2 avec albuminurie > $0.5 \mathrm{~g} / \mathrm{jour}$ ou équivalent. Cent quatre-vingt-huit personnes ont répondu au questionnaire, réparties entre internes (12\%), assistants (13\%), praticiens en hôpital universitaire (26\%), en hôpital général (24\%), en secteur associatif (10\%), en secteur privé (10\%), en exercice mixte (3\%) ou sans pratique clinique (2\%). L'élévation de la protéinurie était un critère d'indication de biopsie rénale pour $51 \%$ des participants, le syndrome néphrotique pour $56 \%$ des participants, l'absence de rétinopathie diabétique pour $57 \%$ des participants, la courte durée du diabète pour $65 \%$ des participants, la progression rapide de l'insuffisance rénale chronique pour $75 \%$ des participants et l'hématurie pour $78 \%$ des participants. Notre enquête révèle donc une incertitude de la communauté néphrologique quant aux critères d'indication de biopsie rénale chez les patients diabétiques de type 2 ayant une protéinurie et la discordance des opinions vis-à-vis des recommandations et de la littérature, en particulier au regard de l'absence de rétinopathie diabétique. Ces résultats soulignent la nécessité d'une étude analysant les résultats de ces biopsies au regard de leurs indications.

Mots clefs: Diabète de type 2, biopsie rénale, néphropathie diabétique, protéinurie, enquête d'opinion 


\section{SUMMARY}

Diabetic nephropathy is usually a presumptive diagnosis based on clinical and biological evidence. Renal biopsies are performed in diabetic patients with atypical findings evoking non diabetic renal disease who could benefit from specific therapies. French speaking nephrologists were asked which criteria they retain to indicate renal biopsy in patients with type 2 diabetes and albuminuria $>0.5 \mathrm{~g} / \mathrm{day}$ or equivalent through an online anonymous questionnaire. Among the suggested criteria were absence of diabetic retinopathy, hematuria, rapid decrease in GFR, short diabetes duration or rapid raise of proteinuria. 188 people answered the poll among whom interns (12\%), fellows (13\%), university hospital practitioners (26\%), general hospital practitioners $(24 \%)$, practitioners in a non-profit organization (13\%), practitioners on private activity $(10 \%)$, multi-modal practitioners (3\%) and people without clinical activity (2\%). Increasing proteinuria was retained as an indication criterion for renal biopsy by $51 \%$ of respondents, nephrotic syndrome by $56 \%$ of respondents, absence of diabetic retinopathy by $57 \%$ of respondents, short diabetes duration by $65 \%$ of respondents, rapid GFR decline by $75 \%$ of respondents and hematuria by $78 \%$ of respondents. These data highlight the high diversity of opinions on this topic and their discrepancies with guidelines and current literature regarding the association between non diabetic renal disease and clinical and biological features. The lack of adhesion of nephrologists to guidelines was especially noteworthy regarding the absence of diabetic retinopathy. These results emphasize the need for studies focusing on biopsy indication criteria in patients with type 2 diabetes.

Keywords: Type 2 diabetes, renal biopsy, diabetic nephropathy, proteinuria, poll 


\section{INTRODUCTION}

L'importante augmentation de la prévalence mondiale du diabète est un sujet de préoccupation majeure. L’Organisation Mondiale de la Santé (OMS) estime qu'il y avait environ 442 millions de personnes diabétiques en 2014, contre 108 millions en 1980 (1). En parallèle, la néphropathie diabétique est devenue la première cause de recours à une technique de suppléance de la fonction rénale en Europe (2) et en Amérique du Nord (3). Ainsi, le nombre de patients diabétiques adressés aux néphrologues à but diagnostique a également augmenté (4). Dans la plupart des cas, le diagnostic de néphropathie diabétique est présomptif, basé sur des arguments cliniques et biologiques, mais seule l'analyse histologique d'une biopsie rénale permet un diagnostic de certitude. Cependant, il s'agit d'un acte invasif comportant un risque d'évènement indésirable sévère, c'est pourquoi le rapport bénéfice/risque de la biopsie rénale chez les patients diabétiques, lorsque le diagnostic de néphropathie diabétique est quasi-certain, est faible (5). En conséquence, c’est la présence d'atypies de présentation qui fait suspecter une maladie rénale non diabétique et peut motiver la réalisation d'une biopsie rénale, permettant un diagnostic de certitude et la prise en charge spécifique du patient. Ces atypies sont définies par les recommandations de la National Kidney Foundation : Kidney Disease Outcomes Quality Initiative (KDOQI) comme (a) l'absence de rétinopathie diabétique, (b) un débit de filtration glomérulaire (DFG) bas ou rapidement décroissant, (c) une élévation rapide de la protéinurie ou syndrome néphrotique, (d) un sédiment urinaire actif, (e) au moins un signe ou symptôme d'une maladie systémique et (f) une hypertension réfractaire et une réduction du DFG $>30 \%$ dans les $2-3$ mois suivant l'initiation d'un antagoniste du système rénine angiotensine (ce dernier critère devant faire rechercher une sténose d'une artère rénale plutôt que de recourir à la biopsie) (6). Une revue récente suggère qu'une biopsie devrait être pratiquée chez les patients diabétiques de type 2 présentant au moins une des atypies définies par les recommandations KDOQI et/ou (g) une ancienneté du diabète inférieure à 5 ans (7). Cette attitude semble justifiée au regard des nombreuses études rétrospectives qui ont mis en évidence l'association d'une maladie rénale non diabétique avec une faible ancienneté du diabète et l'absence de rétinopathie, et, de manière moins constante, avec une hémoglobine glyquée basse $(\mathrm{HbA} 1 \mathrm{c})$, une hématurie et l'absence d'hypertension artérielle (8-14). Nous avons cherché à connaitre le degré d'accord des néphrologues français avec ces 
recommandations, en particulier les points a-d, par un questionnaire en ligne portant sur les éléments cliniques et biologiques pouvant faire porter l'indication de biopsie rénale chez un patient diabétique de type 2 . 


\section{METHODES}

\section{Questionnaire}

Le questionnaire était anonyme. II comportait 26 questions. Les trois premières portaient sur les caractéristiques démographiques du répondeur: type d'exercice, âge et genre. Un court texte de contextualisation suivait : «Chez un patient diabétique de type 2, présentant une albuminurie supérieure à $500 \mathrm{mg} / 24 \mathrm{~h}$ (ou équivalent), lesquels parmi les éléments suivants vous semblent rendre nécessaire la réalisation d'une biopsie rénale pour exclure une maladie rénale non diabétique ?». Les 23 questions suivantes portaient sur des caractéristiques cliniques et biologiques pouvant faire porter l'indication de biopsie rénale. Dix-huit questions étaient posées de façon inconditionnelle et 5 étaient posées en fonction des réponses précédentes; il était demandé de répondre « oui » ou « non » selon que l'indication de biopsie rénale était ou non retenue. Ces questions portaient sur des caractéristiques d'intérêt (atypie ne suggérant pas un diagnostic alternatif particulier) et des caractéristiques confondantes (tableau 1). II était en outre laissé la possibilité d'un commentaire libre en fin de questionnaire. Ces commentaires ont été pris en compte pour corriger les réponses aux questions conditionnelles. Pour soumettre ses réponses au questionnaire, il était nécessaire d'avoir renseigné toutes les réponses à l'exception de l'âge et du genre.

\section{Diffusion du questionnaire et collecte des données}

Le questionnaire a été diffusé par mail par l'intermédiaire de la Société Francophone de Néphrologie, Dialyse et Transplantation et du Club des Jeunes Néphrologues et par mail direct à des néphrologues. Le questionnaire était hébergé par le site www.webquest.fr permettant une collecte des données en ligne.

\section{Analyses statistiques}

Les données ont été analysées et les figures produites avec RStudio integrated development environment (IDE) for $\mathrm{R}(15,16)$ et le package ggplot2 (17). 


\section{RESULTATS}

\section{Participants}

Cent quatre-vingt-huit réponses ont été données au questionnaire. Les caractéristiques démographiques des participants sont résumées dans le tableau 2.

\section{Réponses au questionnaire}

La fréquence de réponses positives aux différentes questions variait de $97 \%$ (L'association d'une maladie auto-immune avec potentielle atteinte rénale) à $31 \%$ (Des anticorps anti-nucléaires sans spécificité connue). Aucune des propositions n'a suscité l'unanimité dans un sens comme dans l'autre.

Parmi les caractéristiques d'intérêt, la présence d'une hématurie, la progression rapide de la maladie rénale chronique et la découverte récente du diabète ont obtenu une distribution des réponses statistiquement différentes de $50 \%$ selon un test du Chi-2 (taux de réponses positives $78 \%, 75 \%$ et $65 \%$ respectivement, $p<$ 0.001 dans les 3 cas ). A l'inverse, les répartitions des réponses pour l'absence de rétinopathie diabétique, le syndrome néphrotique et l'élévation rapide de la protéinurie n'n'étaient pas différentes de $50 \%$ avec un taux de réponses positives à $57 \%(p=0.058), 56 \%(p=0.080)$ et $52 \%(p=0.66)$, traduisant l'absence de réponse majoritaire de la communauté néphrologique dans ces circonstances (Figure 1).

Néanmoins, ces données doivent être mises en regard des réponses aux questions conditionnelles. En effet, si $78 \%$ des néphrologues interrogés considèrent qu'une hématurie chez un patient diabétique est une indication à réaliser une biopsie rénale, $82 \%$ d'entre eux éliminent auparavant une cause urologique et $18 \%$ seulement recourent d'emblée à la biopsie rénale. Des observations similaires peuvent être faites au sujet de l'élévation de la protéinurie et du syndrome néphrotique (Figure 2). Néanmoins, dans ces deux derniers cas, le pourcentage de participants n'ayant pas considéré l'indication de biopsie rénale approche 50\% (tableau 3).

La question sur la découverte récente du diabète relevait d'un autre type de nuance, à savoir la définition du caractère récent de la découverte du diabète. Sur les 122 (65 \%) participants ayant retenu découverte récente du diabète comme une indication de biopsie rénale, les définitions d'une découverte récente variaient de 1 à 
20 ans avec une médiane à 5 ans et une répartition hétérogène des réponses, les 3 plus fréquentes étant 5 ans, 10 ans et 2 ans (respectivement $54 \%, 24 \%$ et $8 \%$ des réponses) (Figure 3 ).

\section{Influence des caractéristiques démographiques sur les critères retenus}

Nous avons analysé si les caractéristiques démographiques avaient une influence sur les réponses. Que ce soit pour le type d'exercice, la classe d'âge ou le genre, la répartition des réponses était globalement homogène à travers toutes les classes. La seule exception notable était celle des quelques participants sans exercice clinique dont les réponses varient de manière importante par rapport aux autres participants. Etant donné la particularité de ces participants et le faible effectif de ce groupe, les analyses statistiques ont été réalisées uniquement à partir des réponses des participants ayant une activité clinique. Dans le détail, il n'y avait aucune différence dans les réponses des participants féminins et masculins. A l'inverse, l'hématurie était retenue comme critère d'indication à une biopsie rénale différemment selon le type d'exercice $(p=0,018)$. En effet, elle était moins fréquemment retenue par les praticiens en exercice mixte (20\%) que par les internes (83\%), les chefs de clinique et assistants (72\%), les praticiens en hôpital universitaire (88\%), en hôpital général (78\%), en secteur associatif $(89 \%)$ ou dans le secteur privé (73\%). La présence d'anticorps antidsDNA et la présence d'anticorps anti-nucléaire (AAN) sans spécificité étaient retenues comme critères d'indication à une biopsie rénale différemment en fonction de l'âge des néphrologues. En effet, la présence d'anti-dsDNA était moins souvent $(p=0,010)$ retenue comme indication chez les $40-60$ ans $(66 \%)$ que chez les moins de 30 ans (79\%), les 30-40 ans (88\%) et les plus de 60 ans (90\%). En parallèle, la présence d'AAN sans spécificité était plus souvent $(p=0,044)$ retenue comme indication chez les plus de 60 ans $(55 \%)$ que chez les moins de 30 ans (29\%), les $30-40$ ans (30\%) et les $40-60$ ans (25\%). On constate cependant que ces différences sont minimes et témoignent d'une grande homogénéité dans les réponses à travers les types d'exercice, l'âge et le genre (Figure 4).

\section{Commentaires}


Vingt-six participants ont exprimé un commentaire. Il était fréquemment souligné que les situations cliniques réelles sont généralement plus complexes et nuancées que ce que permet un questionnaire. D'autres critères pertinents ont également été suggérés pour 1) contextualiser la situation : terrain (âge, genre, antécédents vasculaires), qualité des équilibres glycémique et tensionnel, 2) pour eux-mêmes : antécédent familial de maladie rénale, néphrotoxicité des médicaments, présence d'anti-phospholipides ou 3) pour nuancer ceux déjà proposés : profondeur de l'hypoalbuminémie pour le syndrome néphrotique et élimination d'une sténose de l'artère rénale en cas de progression rapide de la maladie rénale chronique. Il a enfin été rappelé que quelle que soit l'indication, la décision de réaliser la biopsie rénale devait tenir compte du rapport bénéfice/risque. 


\section{DISCUSSION}

Notre enquête menée auprès de 188 néphrologues, basée sur le volontariat a mis en évidence un certain degré d'incertitude de la communauté néphrologique quant aux critères d'atypie qui justifient une biopsie rénale chez les patients diabétiques de type 2 avec une albuminurie supérieure à $0,5 \mathrm{~g} / 24 \mathrm{heures}$. Cela était d'autant plus vrai pour les atypies sans orientation étiologique propre telles qu'un syndrome néphrotique, une élévation de la protéinurie, l'absence de rétinopathie diabétique et une faible ancienneté du diabète en dépit de recommandations et d'une littérature incitant à considérer ces atypies sans orientation étiologique propre, comme devant faire douter du diagnostic de néphropathie diabétique et donc à réaliser une biopsie rénale qui expliquent probablement l'augmentation de la part de néphropathie diabétique parmi les diagnostics portés sur les biopsies rénales durant les 30 dernières années (4). Cependant, les études sur le sujet n’ont généralement pas analysé les indications de biopsie rénale, mais l'association de caractéristiques cliniques avec le diagnostic de maladie rénale non diabétique.

Les résultats de l'enquête sont en discordance avec des données de la littérature et ce, de manière relativement homogènes, indépendamment du genre ou de l'âge. Seule l'hématurie semblait être prise en compte différemment en fonction du mode d'exercice, mais cette discordance est à interpréter avec prudence étant donné le faible effectif du groupe des praticiens en exercice mixte. En effet, si la présence d'une hématurie est l'atypie la plus constamment associée à une maladie rénale non diabétique dans la littérature, l'absence de rétinopathie diabétique est la seconde et pourtant cette indication est loin de faire consensus au sein de la communauté néphrologique francophone. Il faut cependant noter que ces études portaient sur les caractéristiques cliniques associées au diagnostic de maladie rénale non diabétique et seules de rares études ont considéré les indications de biopsie rénale en tant que telles (8-10). Ainsi, la prise en compte dans une étude «classique » d'un patient diabétique de type 2 protéinurique mais sans rétinopathie «biopsié » pour une insuffisance rénale aiguë de cause non évidente et présentant une nécrose tubulaire aiguë renforcerait l'association de l'absence de rétinopathie diabétique avec le diagnostic de maladie rénale non diabétique alors même que cette caractéristique clinique ne fonde pas la décision de réaliser une biopsie rénale. C'est probablement cette discordance entre l'objectif de ces études (aider le clinicien dans sa décision face au patient) et leur méthodologie qui explique la division de notre communauté face à leurs conclusions. 
A l'inverse, la répartition des réponses concernant la découverte récente du diabète est en adéquation avec les données contradictoires de la littérature. En effet, sur 18 études $(8,10,18-33)$ qui ont étudié l'ancienneté de la découverte du diabète comme prédicteur d'une maladie rénale non diabétique sans exclure les patients ayant une maladie rénale mixte (diabétique et non diabétique), dix (56\%) n'ont pas montré d'association entre cette caractéristique clinique et la présence d'une maladie rénale non diabétique. Ces variations sont probablement liées à la latence diagnostic du diabète de type II puisqu'il est estimé que la moitié des patients diabétique de type 2 ont déjà des complications au moment du diagnostic (34). Ainsi, il est recommandé de dépister la néphropathie diabétique par une recherche de microalbuminurie dès le diagnostic chez les patients diabétiques de type 2 et après 5 ans d'évolution chez les patients diabétiques de type 1, le diabète s'installant plus bruyamment dans ce cas entrainant un diagnostic contemporain (6). C'est cette difficulté à dater un diabète de type 2 qui explique pourquoi le critère temporel n'avait pas été retenu dans les recommandations KDOQI. Cette difficulté est d'ailleurs illustrée par les réponses multiples faites par les participants à la présente étude pour la définition d'un diabète de diagnostic récent. Ce n'est que plus tard qu'un diagnostic récent de diabète a émergé comme indication à une biopsie rénale chez les patients diabétiques de type 2 en raison d'une ancienneté plus importante de diagnostic de diabète de type 2 chez les patients avec une néphropathie diabétique isolée que chez les patients avec une maladie rénale non diabétique avec ou sans néphropathie diabétique. Là encore, ce sont les différences concernant la caractéristique clinique entre les 2 situations diagnostiques qui ont été comparées plutôt que la fréquence des situations diagnostiques dans la population de patients chez qui la biopsie a été faite en raison de la découverte récente du diabète. II est cependant à noter que lorsque l'ancienneté du diabète est réellement connue du fait d'un dépistage systématique ou de résultats biologiques antérieurs normaux, l'association entre l'ancienneté du diabète et la probabilité de survenue d'une néphropathie diabétique semble aussi importante dans le diabète de type 2 que dans le diabète de type 1 (35).

De manière générale, les recommandations KDOQI proprement dites ne distinguent pas diabète de type 1 et diabète de type 2 en ce qui concerne la recherche d'une autre maladie rénale. Ce n'est que dans l'argumentaire que des nuances sont apportées, en particulier concernant la valeur de la présence d'une rétinopathie diabétique. II y est en effet précisé que chez les patients « macroalbuminurique » (> $300 \mathrm{mg} / \mathrm{g}$ ) la 
valeur prédictive négative de la rétinopathie diabétique pour la présence d’une néphropathie diabétique varie entre 20 et $84 \%$ selon les études. L'absence de rétinopathie diabétique n'est donc pas synonyme d'absence de néphropathie diabétique. A l'inverse, la valeur prédictive positive varie entre 67 et $100 \%$. La présence d'une rétinopathie diabétique suggère donc la présence d'une néphropathie diabétique. Elle ne suffit cependant pas à éliminer la suspicion d'une autre maladie rénale en présence d'une autre atypie. II faut ajouter enfin que les sujets atteints de diabète de type 2 sont généralement plus âgées au diagnostic que ceux atteints de diabète de type 1 et donc plus susceptibles d'être atteints de pathologies multiples. Si dans le cas du diabète de type 1 affirmer, sur des arguments cliniques, la néphropathie diabétique permet d'exclure une maladie rénale non diabétique du fait de la faible probabilité d'une maladie rénale autre à un jeune âge, ce raisonnement est peut-être moins juste pour les patients atteints de diabète de type 2 plus susceptibles d'avoir une maladie rénale non diabétique associée.

Cette enquête présente un certain nombre de limitations. L'effet centre n'a pas été étudié. Cette omission volontaire a été motivée par la volonté de maintenir l'anonymat des données démographiques collectées. II est possible que la présentation conditionnelle des propositions nuancées ait augmenté le nombre de néphrologues ne considérant pas le recours à la biopsie dans certaines situations, comme la présence d'un syndrome néphrotique. Néanmoins, il nous a semblé important de ne pas proposer d'emblée ces nuances. En effet, comme relevé dans de nombreux commentaires, le choix de retenir une indication de biopsie rénale est rarement dichotomique, et une «voie médiane » aurait probablement remporté l'adhésion unanime des participants au questionnaire. Enfin, la simplicité relative du questionnaire ne permettait pas une prise en compte adéquate du facteur temps. Or, la pratique clinique repose en grande partie sur l'histoire du patient. II est d'ailleurs à noter que les critères intégrant la dimension temporelle tels que la progression « trop rapide » de la dysfonction rénale et l'augmentation «trop rapide » de la protéinurie sont subjectifs et en l'absence de définition claire dans les recommandations laissées à la totale appréciation du clinicien.

En dépit de ces limites, notre enquête met en lumière l'incertitude de la communauté et souligne sur la nécessité d'une étude analysant la rentabilité des critères d'atypie comme indications de biopsies rénales chez les patients diabétiques au regard du diagnostic de maladie rénale non diabétique. 
CONFLITS D'INTERET : E.D. a reçu des émoluments personnels de la part d'ASTRA ZENECA, FRESENIUS MEDICAL CARE, VIFOR/ Fresenius Medical Care et SANOFI, sans rapport avec le présent travail. F.V. a reçu des émoluments personnels de la part de FRESENIUS MEDICAL CARE, et BAXTER sans rapport avec le présent travail. C.V a reçu des émoluments personnels de la part d'Amicus, astellas et VIFOR/ Fresenius Medical Care sans rapport avec le présent travail. J.M.C. a reçu une aide non financière de la part d'ALEXION, sans rapport avec le présent travail. Les résultats présentés dans ce manuscrit ont été présentés sous forme de poster au 3e congrés de la SFNDT, Lille, 2018.

FINANCEMENT : Cette étude n'a pas reçu de soutien financier de la part d'une entité publique, privée ou associative.

REMERCIEMENTS : Les auteurs remercient tous les participants à l'étude d'avoir pris le temps de répondre au questionnaire et la SFNDT et le CJN pour avoir diffusé l'enquête auprès de leurs membres.

CONTRIBUTION DES AUTEURS : Jonathan M Chemouny a conceptualisé l'étude et sa méthodologie, collecté et analysé les données et écrit le manuscrit; Aurélie Sannier, Guillaume Hanouna, Quentin Raimbourg, Eric Daugas et Cécile Vigneau ont relu et corrigé le manuscrit ; Francois Vrtovnsik a supervisé l'étude, validé les résultats et écrit le manuscrit 


\section{REFERENCES}

1. NCD Risk Factor Collaboration (NCD-RisC). Worldwide trends in diabetes since 1980: a pooled analysis of 751 population-based studies with 4.4 million participants. Lancet Lond Engl. 2016 Apr 9;387(10027):1513-30.

2. Pippias M, Kramer A, Noordzij M, Afentakis N, Alonso de la Torre R, Ambühl PM, et al. The European Renal Association - European Dialysis and Transplant Association Registry Annual Report 2014: a summary. Clin Kidney J [Internet]. 2017 Jan 16 [cited 2017 Mar 21]; Available from:

https://academic.oup.com/ckj/ckj/article/2978059/The

3. Chapter 1: Incidence, Prevalence, Patient Characteristics, and Treatment Modalities. Am J Kidney Dis. 67(3):S13958.

4. O'Shaughnessy MM, Hogan SL, Poulton CJ, Falk RJ, Singh HK, Nickeleit V, et al. Temporal and Demographic Trends in Glomerular Disease Epidemiology in the Southeastern United States, 1986-2015. Clin J Am Soc Nephrol. 2017 Mar 21;CJN.10871016.

5. Dhaun N, Bellamy CO, Cattran DC, Kluth DC. Utility of renal biopsy in the clinical management of renal disease. Kidney Int. 2014 May;85(5):1039-48.

6. KDOQI Clinical Practice Guidelines and Clinical Practice Recommendations for Diabetes and Chronic Kidney Disease. Am J Kidney Dis. 2007 Feb;49(2):S12-154.

7. Doshi SM, Friedman AN. Diagnosis and Management of Type 2 Diabetic Kidney Disease. Clin J Am Soc Nephrol CJASN. 2017 Mar 9;

8. Bermejo S, Soler MJ, Gimeno J, Barrios C, Rodríguez E, Mojal S, et al. Predictive factors for non-diabetic nephropathy in diabetic patients. The utility of renal biopsy. Nefrol Engl Ed [Internet]. 2016 [cited 2017 Mar 11]; Available from: http://www.sciencedirect.com/science/article/pii/S2013251416301250

9. Soni SS, Gowrishankar S, Kishan AG, Raman A. Non diabetic renal disease in type 2 diabetes mellitus. Nephrol Carlton Vic. 2006 Dec;11(6):533-7.

10. Mami I, Harzallah A, Kaaroud H, Aoudia R, Hamida FB, Goucha R, et al. Nondiabetic renal disease in patients with type 2 diabetes. Saudi J Kidney Dis Transplant Off Publ Saudi Cent Organ Transplant Saudi Arab. 2017 Aug;28(4):842-50.

11. Lee YH, Kim K-P, Kim YG, Moon J-Y, Jung SW, Park E, et al. Clinicopathological features of diabetic and nondiabetic renal diseases in type 2 diabetic patients with nephrotic-range proteinuria. Medicine (Baltimore). 2017 Sep;96(36):e8047.

12. Zhou J, Chen X, Xie Y, Li J, Yamanaka N, Tong X. A differential diagnostic model of diabetic nephropathy and nondiabetic renal diseases. Nephrol Dial Transplant Off Publ Eur Dial Transpl Assoc - Eur Ren Assoc. 2008 Jun;23(6):1940-5.

13. Liang S, Zhang X-G, Cai G-Y, Zhu H-Y, Zhou J-H, Wu J, et al. Identifying Parameters to Distinguish Non-Diabetic Renal Diseases from Diabetic Nephropathy in Patients with Type 2 Diabetes Mellitus: A Meta-Analysis. Najafian B, editor. PLoS ONE. 2013 May 14;8(5):e64184.

14. Fiorentino M, Bolignano D, Tesar V, Pisano A, Biesen WV, Tripepi G, et al. Renal biopsy in patients with diabetes: a pooled meta-analysis of 48 studies. Nephrol Dial Transplant Off Publ Eur Dial Transpl Assoc - Eur Ren Assoc. 2017 01;32(1):97-110. 
15. RStudio Team. RStudio: Integrated Development Environment for R [Internet]. Boston, MA: RStudio, Inc.; 2016. Available from: http://www.rstudio.com/

16. R Core Team. R: A Language and Environment for Statistical Computing [Internet]. Vienna, Austria: R Foundation for Statistical Computing; 2017. Available from: https://www.R-project.org/

17. Wickham H. ggplot2: Elegant Graphics for Data Analysis [Internet]. Springer-Verlag New York; 2009. Available from: http://ggplot2.org

18. Tone A, Shikata K, Matsuda M, Usui H, Okada S, Ogawa D, et al. Clinical features of non-diabetic renal diseases in patients with type 2 diabetes. Diabetes Res Clin Pract. 2005 Sep;69(3):237-42.

19. Bi H, Chen N, Ling G, Yuan S, Huang G, Liu R. Nondiabetic renal disease in type 2 diabetic patients: a review of our experience in 220 cases. Ren Fail. 2011;33(1):26-30.

20. Lin Y-L, Peng S-J, Ferng S-H, Tzen C-Y, Yang C-S. Clinical indicators which necessitate renal biopsy in type 2 diabetes mellitus patients with renal disease. Int J Clin Pract. 2009 Aug;63(8):1167-76.

21. Suzuki D, Takano H, Toyoda M, Umezono T, Uehara G, Sakai T, et al. Evaluation of renal biopsy samples of patients with diabetic nephropathy. Intern Med Tokyo Jpn. 2001 Nov;40(11):1077-84.

22. Chang TI, Park JT, Kim J, Kim SJ, Oh HJ, Yoo DE, et al. Renal outcomes in patients with type 2 diabetes with or without coexisting non-diabetic renal disease. Diabetes Res Clin Pract. 2011 May;92(2):198-204.

23. Byun JM, Lee CH, Lee SR, Moon JY, Lee SH, Lee TW, et al. Renal outcomes and clinical course of nondiabetic renal diseases in patients with type 2 diabetes. Korean J Intern Med. 2013 Sep;28(5):565-72.

24. Kritmetapak K, Anutrakulchai S, Pongchaiyakul C, Puapairoj A. Clinical and pathological characteristics of nondiabetic renal disease in type 2 diabetes patients. Clin Kidney J. 2018 Jun 1;11(3):342-7.

25. Wilfred DC, Mysorekar VV, Venkataramana RS, Eshwarappa M, Subramanyan R. Nondiabetic Renal Disease in type 2 Diabetes Mellitus Patients: A Clinicopathological Study. J Lab Physicians. 2013 Jul;5(2):94-9.

26. Horvatic I, Tisljar M, Kacinari P, Matesic I, Bulimbasic S, Galesic Ljubanovic D, et al. Non-diabetic renal disease in Croatian patients with type 2 diabetes mellitus. Diabetes Res Clin Pract. 2014 Jun;104(3):443-50.

27. Mak SK, Gwi E, Chan KW, Wong PN, Lo KY, Lee KF, et al. Clinical predictors of non-diabetic renal disease in patients with non-insulin dependent diabetes mellitus. Nephrol Dial Transplant Off Publ Eur Dial Transpl Assoc - Eur Ren Assoc. 1997 Dec;12(12):2588-91.

28. Pallayova M, Mohammed A, Langman G, Taheri S, Dasgupta I. Predicting non-diabetic renal disease in type 2 diabetic adults: The value of glycated hemoglobin. J Diabetes Complications. 2015 Jul;29(5):718-23.

29. Akimoto T, Ito C, Saito O, Takahashi H, Takeda S, Ando Y, et al. Microscopic hematuria and diabetic glomerulosclerosis--clinicopathological analysis of type 2 diabetic patients associated with overt proteinuria. Nephron Clin Pract. 2008;109(3):c119-126.

30. Erdogmus S, Kiremitci S, Celebi ZK, Akturk S, Duman N, Ates K, et al. Non-Diabetic Kidney Disease in Type 2 Diabetic Patients: Prevalence, Clinical Predictors and Outcomes. Kidney Blood Press Res. 2017;42(5):886-93.

31. Sharma SG, Bomback AS, Radhakrishnan J, Herlitz LC, Stokes MB, Markowitz GS, et al. The Modern Spectrum of Renal Biopsy Findings in Patients with Diabetes. Clin J Am Soc Nephrol. 2013 Oct 7;8(10):1718-24. 
32. Ghani AA, Al Waheeb S, Al Sahow A, Hussain N. Renal biopsy in patients with type 2 diabetes mellitus: indications and nature of the lesions. Ann Saudi Med. 2009 Dec;29(6):450-3.

33. Lee $\mathrm{EY}$, Chung $\mathrm{CH}$, Choi SO. Non-diabetic renal disease in patients with non-insulin dependent diabetes mellitus. Yonsei Med J. 1999 Aug;40(4):321-6.

34. Intensive blood-glucose control with sulphonylureas or insulin compared with conventional treatment and risk of complications in patients with type 2 diabetes (UKPDS 33). The Lancet. 1998 Sep 12;352(9131):837-53.

35. Nelson RG, Newman JM, Knowler WC, Sievers ML, Kunzelman CL, Pettitt DJ, et al. Incidence of end-stage renal disease in type 2 (non-insulin-dependent) diabetes mellitus in Pima Indians. Diabetologia. 1988 Oct;31(10):730-6. 


\section{Tableau 1}

\section{Questions d'intérêt}

L'absence de rétinopathie diabétique?

La présence d'une hématurie?

La progression rapide de l'insuffisance rénale (définie par une augmentation de la créatininémie d'au moins $50 \%$ progressivement sur plusieurs prélèvements successifs et sur plusieurs mois) ?

\section{L'élévation rapide de la protéinurie?}

Un syndrome néphrotique?

Une découverte récentediabète?

\section{Questions confondantes}

Une néoplasie connue?

L'association d'une maladie auto-immune avec potentielle atteinte rénale ?

Des anticorps anti-nucléaires sans spécificité connue?

Des ANCA non anti-MPO, non anti-PR3 ?

Des anticorps anti-nucléaires anti-ADN natif (anti-dsDNA) sans manifestation extra-rénale?

Des ANCA anti-MPO ou anti-PR3 sans manifestation extra-rénale ?

Une gammapathie monoclonale?

Une insuffisance rénale aiguë (définie par une augmentation de la créatininémie d'au moins $50 \%$ sur deux prélèvements successifs en moins de 3 mois sans cause évidente) ?

Une sérologie positive pour les VIH, VHB et/ou VHC?

Y compris en l'absence de réplication virale?

L'existence d'une protéinurie supérieure à $500 \mathrm{mg} / 24 \mathrm{~h}$ documentée avant le diagnostic de diabète?

Un purpura vasculaire?

Une (ou des) manifestation(s) clinique(s) pouvant entrer dans le cadre d'une maladie systémique avec atteinte rénale sans diagnostic posé ? 
n (\%)

\section{Type d'exercice}

Interne

$23(12 \%)$

Chef de clinique/assistant

$25(13 \%)$

Praticien en hôpital universitaire

$48(26 \%)$

Praticien en hôpital général

$45(24 \%)$

$19(10 \%)$

Praticien dans le secteur associatif

$5(3 \%)$

Praticien en exercice mixte

$19(10 \%)$

Praticien dans le secteur privé

$4(2 \%)$

\section{Tranches d'âge}

Moins de 30 ans

$24(13 \%)$

Entre 30 et 40 ans

$79(42 \%)$

Entre 40 et 60 ans

$64(34 \%)$

Plus de 60 ans

$20(11 \%)$

Non renseignée

$1(1 \%)$

Féminin

$76(40 \%)$

Masculin

$104(55 \%)$

Non renseignée

$8(4 \%)$ 
Indication de biopsie rénale

\begin{tabular}{|c|c|c|c|c|}
\hline \multirow[b]{2}{*}{ Question } & \multicolumn{3}{|c|}{ (2) } & \multirow[b]{2}{*}{ Conditions } \\
\hline & $\begin{array}{l}\text { Jamais } \\
\text { retenue }\end{array}$ & $\begin{array}{l}\text { Retenue sous } \\
\text { condition }\end{array}$ & $\begin{array}{l}\text { Systématiquement } \\
\text { retenue }\end{array}$ & \\
\hline L'élévation rapide de la protéinurie & $48 \%$ & $35 \%$ & $16 \%$ & $\begin{array}{l}\text { En dehors d'un contexte } \\
\text { d'arrêt du blocage du SRA }\end{array}$ \\
\hline Un syndrome néphrotique & $44 \%$ & $38 \%$ & $18 \%$ & En cas d'apparition brutale \\
\hline Absence de rétinopathie diabétique & $43 \%$ & & $57 \%$ & \\
\hline La découverte récente du diabète & $35 \%$ & & $65 \%$ & \\
\hline $\begin{array}{l}\text { La progression rapide de l'insuffisance } \\
\text { rénale chronique }\end{array}$ & $25 \%$ & & $75 \%$ & \\
\hline Présence d'une hématurie & $22 \%$ & $64 \%$ & $14 \%$ & $\begin{array}{l}\text { Après élimination d'une } \\
\text { cause urologique }\end{array}$ \\
\hline
\end{tabular}




\section{Figure 1 : Répartition des réponses dans la cohorte totale}

\section{Répartitions des réponses dans la cohorte totale}

La présence d'une maladie auto-immune avec potentielle atteinte rénale Un (ou des) élément(s) évocateur(s) d'une maladie systémique

Des lésions purpuriques Une insuffisance rénale aigue La présence d'ANCA anti-MPO ou anti-PR3 La présence d'anti-dsDNA

Présence d'une hématurie * La présence d'une gammapathie monoclonale La progression rapide de I insuffisance rénale chronique *

Une sérologie virale positive Une protéinurie documentée avant le diagnostic du diabète La durée courte du diabète * Absence de rétinopathie diabétique

Un syndrome néphrotique * élévation rapide de la protéinurie La présence d'ANCA non anti-MPO ou anti-PR3

La présence d'une néoplasie La présence de FAN sans spécificité

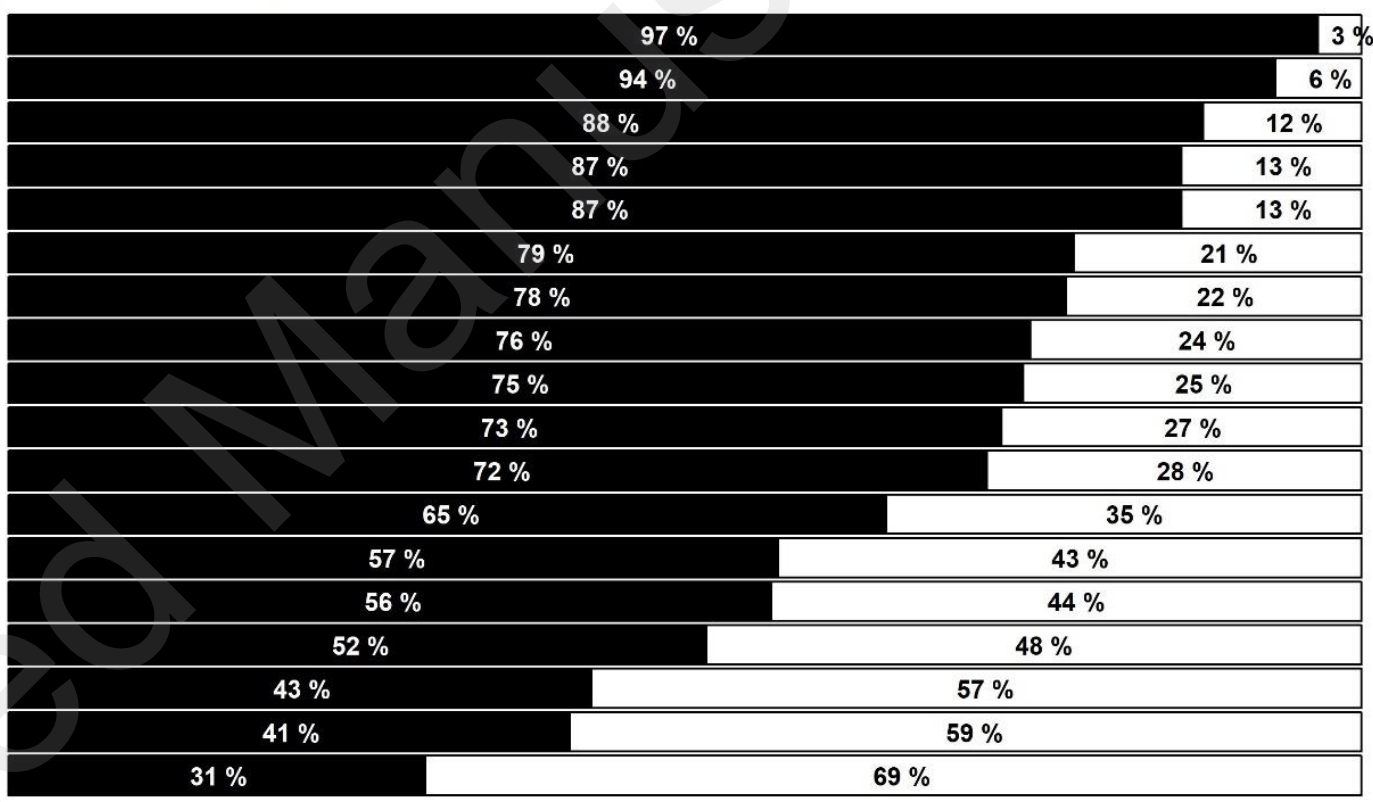


Figure 2 : Répartition des réponses aux questions conditionnelles

Une sérologie virale positive En cas de réplication virale $(n=140)$

Un syndrome néphrotique En cas d'apparition brutale $(n=107)$

$\mathrm{L}$ élévation rapide de la protéinurie En dehors d'un contexte d'arrêt du blocage du SRA $(n=99)$

Présence d'une hématurie Après élimination d'une cause urologique $(n=149)$

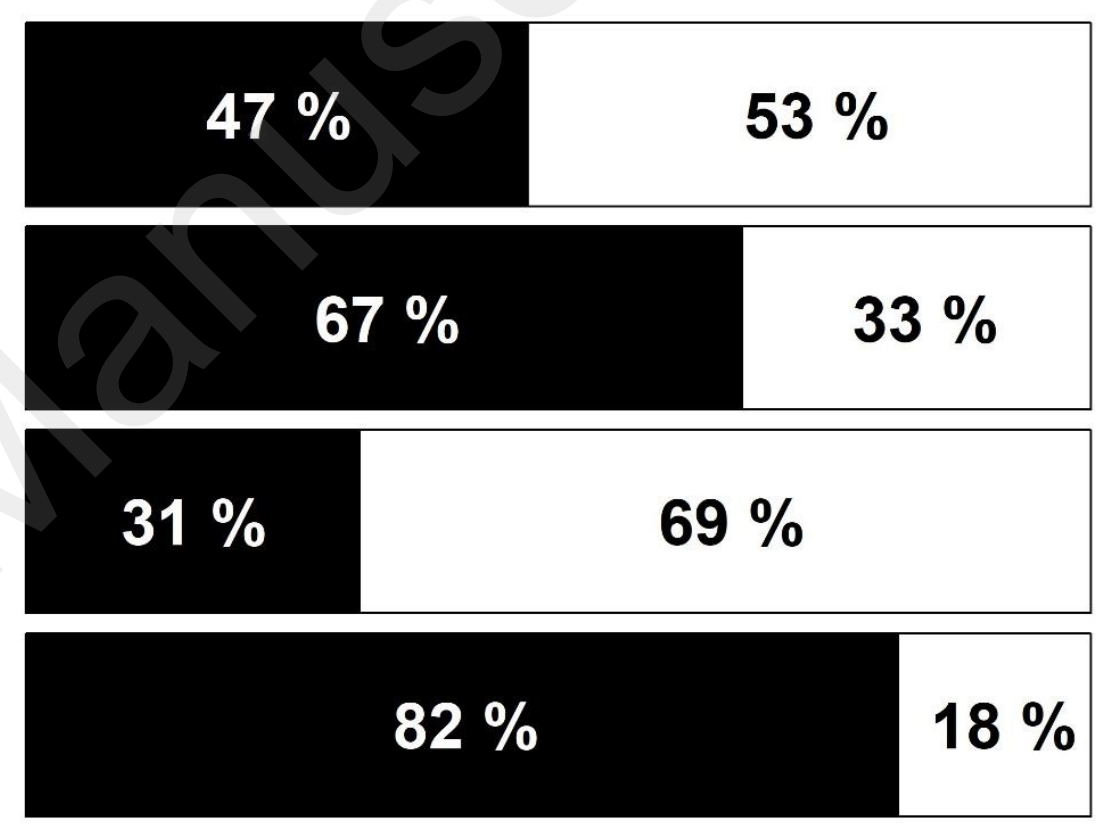

Réponses aux conditions $\square$ Oui $\square$ Non 
Figure 3 : Seuils retenus comme une découverte récente du diabète

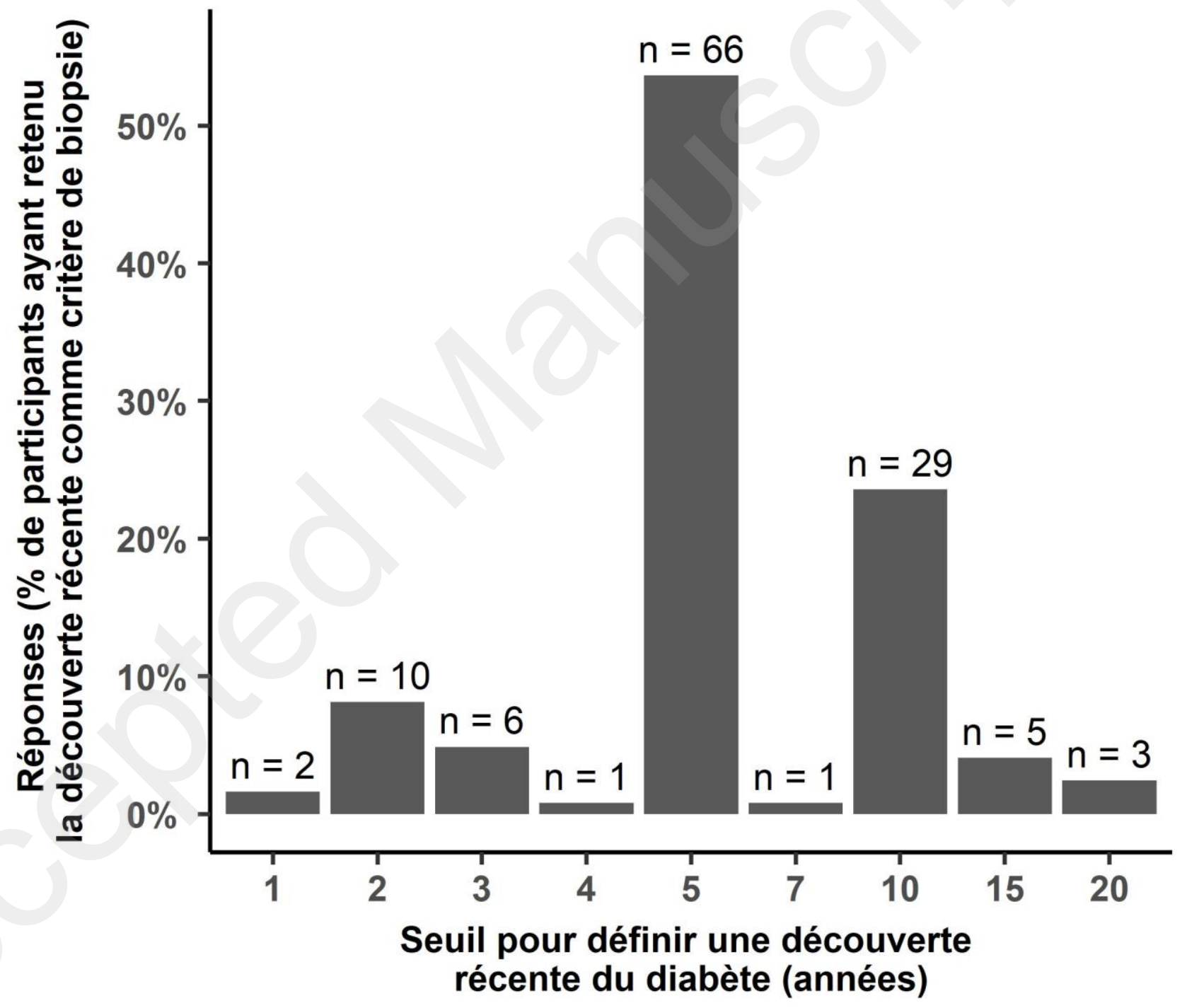


Figure 4 : Répartition des réponses en fonction des modalités d'exercice et des caractéristiques démographiques (l'ordre des items est superposable à celui de la figure 1)

Répartitions des réponses dans la cohorte : par type d'exercice
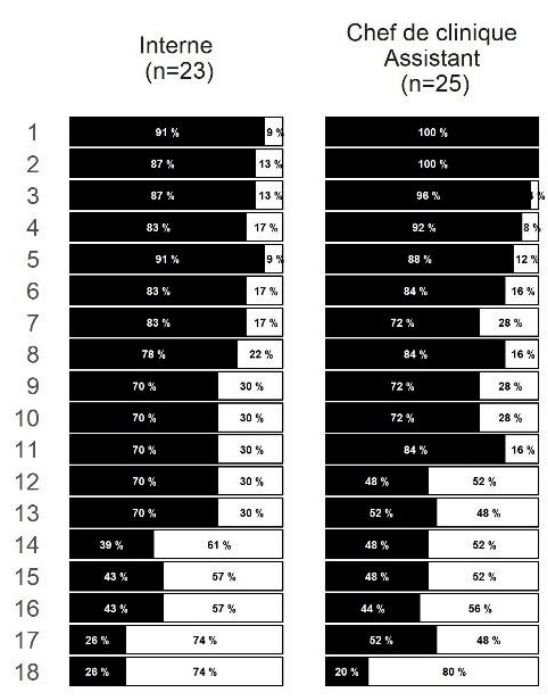

En CHU

En CHG

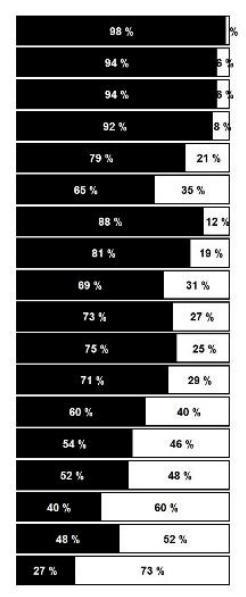

Dans l'associatif

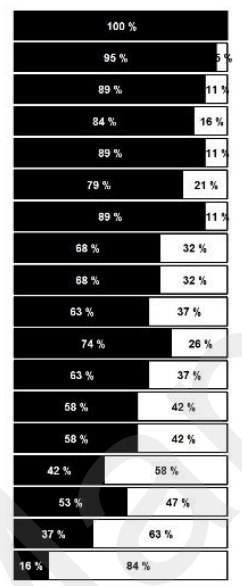

par classe d'âge

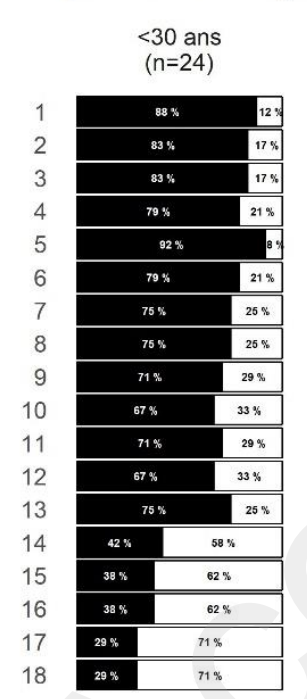

$30-40$ ans
$(n=79)$

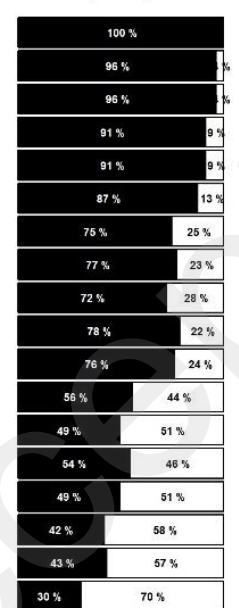

40-60 ans

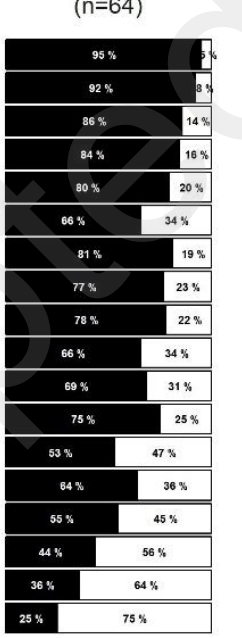

$>60$ ans

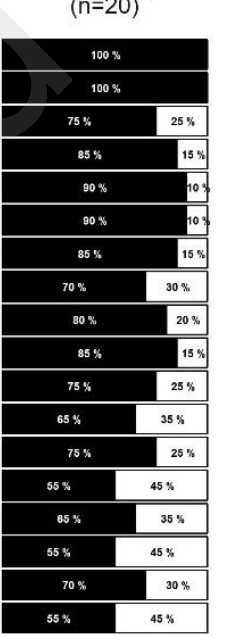

Exercice mixte

$(n=5)$

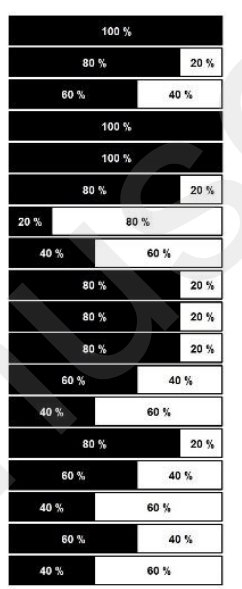

En privé

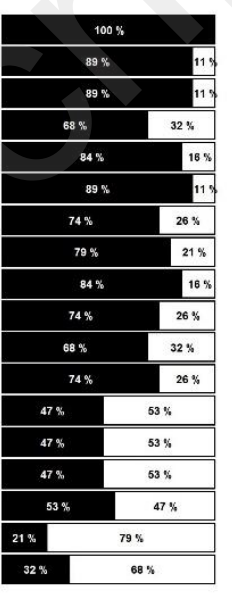

Non clinque

$(n=4)$

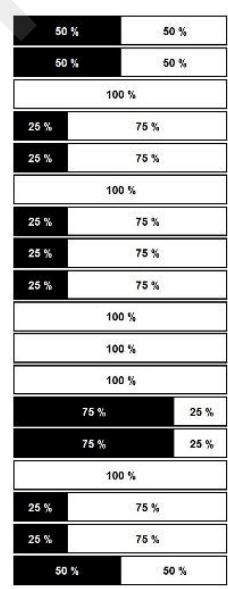

Indication de biopsie retenue $\square$ Oui $\square$ Non

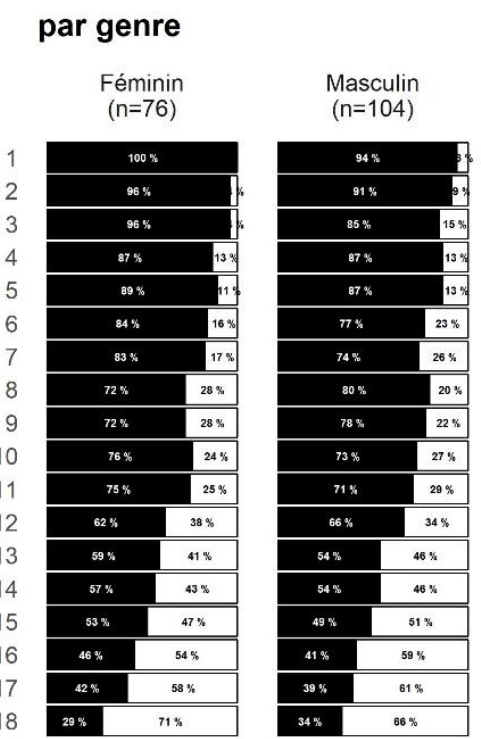

